PROCEEDINGS OF THE

AMERICAN MATHEMATICAL SOCIETY

Volume 127, Number 2, February 1999, Pages 599-608

S 0002-9939(99)04541-4

\title{
THE CENTRAL LIMIT THEOREM FOR LACUNARY SERIES
}

\author{
KATUSI FUKUYAMA AND SHIGERU TAKAHASHI
}

(Communicated by Stanley Sawyer)

\begin{abstract}
In this paper, the central limit theorem for lacunary trigonometric series is proved. Two gap conditions by Erdős and Takahashi are extended and unified. The criterion for the Fourier character of lacunary series is also given.
\end{abstract}

\section{INTRODUCTION}

It is well known that the lacunary trigonometric series $\sum a_{i} \cos \left(2 \pi n_{i} \omega+\phi_{i}\right)$ behaves like random series when $\left\{n_{i}\right\}$ increases very fast. For example, if $\left\{n_{i}\right\}$ has Hadamard gaps, i.e. $n_{i+1} / n_{i}>q>1$, the series converges or diverges almost everywhere according as $\sum a_{i}^{2}$ converges or diverges. (Kolmogorov [3] and Zygmund [10].) It is also known that the series is not a Fourier series of integrable function when $\sum a_{i}^{2}=\infty$. (Zygmund [10].)

As to the central limit theorem for the series with Hadamard gaps, SalemZygmund [4] proved: If $A_{n}=\left(\frac{1}{2} \sum_{i=1}^{n} a_{i}^{2}\right)^{1 / 2} \rightarrow \infty$ and $a_{n}=o\left(A_{n}\right)$ are satisfied, then

$$
\frac{1}{A_{n}} \sum_{i=1}^{n} a_{i} \cos \left(2 \pi n_{i} \omega+\phi_{i}\right) \stackrel{\mathcal{D}}{\longrightarrow} N_{0,1}
$$

holds on probability space $(\Omega, d \omega /|\Omega|)$, when $\Omega \subset[0,1]$ has positive measure. Here $|\cdot|$ denotes Lebesgue measure, $N_{0,1}$ the standard normal distribution, and $\stackrel{\mathcal{D}}{\longrightarrow}$ convergence in law.

Erdős [1] relaxed the gap condition to

$$
n_{i+1} / n_{i}>1+c_{i} / \sqrt{i} \text { where } c_{i} \rightarrow \infty,
$$

and proved (1.1) for $a_{n} \equiv 1$. Takahashi [6] proved that $a_{n} \equiv 1$ can be relaxed to

$$
A_{n} \rightarrow \infty \text { and } a_{n}=O\left(A_{n} / \sqrt{n}\right) .
$$

Takahashi [7] also proved (1.1) assuming

$$
n_{i+1} / n_{i}>1+c / i^{\alpha}, \quad A_{n} \rightarrow \infty \text { and } a_{n}=o\left(A_{n} / n^{\alpha}\right),
$$

where $c>0$ and $0 \leq \alpha \leq 1 / 2$. It should be noted that there is no implication between (1.2) and (1.3), and (1.4). Indeed, if we put $\alpha=1 / 2$, the gap condition

Received by the editors January 27, 1997 and, in revised form, May 23, 1997.

1991 Mathematics Subject Classification. Primary 60F05, 42A55.

Key words and phrases. Lacunary series, central limit theorem, Fourier series, Fourier Stieltjes series.

(C)1999 American Mathematical Society 
of (1.4) is weaker than (1.2), but if we put $a_{n} \equiv 1$, we must put $\alpha<1 / 2$ in (1.4), which is stronger than (1.2).

From (1.1), by the way, we can deduce that the series is not a Fourier series. Therefore, the series is not a Fourier series under (1.4). Takahashi [8] proved that this claim remains valid even if we relax $a_{n}=o\left(A_{n} / n^{\alpha}\right)$ to $a_{n}=O\left(A_{n} / n^{\alpha}\right)$. Under this condition, (1.1) does not hold generally. A counterexample was constructed by Takahashi [9]. Previously, Erdős [1] had noted the existence of such an example for $\alpha=1 / 2$.

Although these results have been considered to be best possible, we still have the following examples excluded from the above scheme: Under the conditions

$$
n_{i+1} / n_{i}>1+c / \sqrt{i \log i}, \quad A_{n} \rightarrow \infty \quad \text { and } \quad a_{n}=o\left(A_{n} / \sqrt{n \log n}\right),
$$

the central limit theorem (1.1) holds. Even if we relax the last condition to $a_{n}=$ $O\left(A_{n} / \sqrt{n \log n}\right)$, the series is not a Fourier series, but there are counterexamples for (1.1).

In this note we introduce a more general gap condition and prove theorems including all the above results and examples.

Theorem 1. Let us suppose the following conditions:

$$
\begin{aligned}
& \lambda(i)>\lambda \text { for some } \lambda>0 ; \\
& \lambda(i+1)-\lambda(i)=o(1) ; \\
& n_{i+1} / n_{i}>1+c / \lambda(i) \text { for some } c>0 ; \\
& a_{n}=o\left(A_{n} / \lambda(n)\right) \text { and } A_{n} \rightarrow \infty .
\end{aligned}
$$

Then (1.1) holds on any $\Omega \subset[0,1]$ with positive measure.

In Theorem 1, the next condition is implicitly assumed:

$$
\sum_{i=1}^{\infty} \frac{1}{\lambda^{2}(i)}=\infty .
$$

Or, more precisely, the existence of $\left\{a_{i}\right\}$ satisfying (1.8) is equivalent to (1.9). Indeed, if (1.9) is false, the contradiction $A_{n}^{2}=O\left(\frac{1}{2} \sum_{i=1}^{n} A_{i}^{2} / \lambda^{2}(i)\right)=o\left(A_{n}^{2}\right)$ follows. In case (1.9) is valid, we can construct $\left\{a_{i}\right\}$ satisfying (1.8), by putting $A_{n}=\exp \left(\frac{1}{2} \sum_{i=1}^{n} e_{i}\right)$ and $a_{n}^{2}=\left(A_{n}^{2}-A_{n-1}^{2}\right) / 2$, where $\left\{e_{i}\right\}$ satisfies $0<e_{i}=$ $o\left(1 / \lambda^{2}(i)\right)$ and $\sum_{i=1}^{\infty} e_{i}=\infty$.

Although Theorem 1 contains assumptions that generalize (1.4), we can derive the next corollary which assumes the generalization of (1.2) and (1.3). The condition (1.9) is again implicitly assumed for $\{\Lambda(i)\}$.

Corollary. Let us suppose the following conditions:

$$
\begin{aligned}
& \Lambda(i)>0 \quad \text { and } \quad \Lambda(i) \rightarrow \infty \\
& \Lambda(i+1)-\Lambda(i)=O(1) ; \\
& n_{i+1} / n_{i}>1+c_{i} / \Lambda(i) \text { where } \quad c_{i} \rightarrow \infty \\
& a_{n}=O\left(A_{n} / \Lambda(n)\right) \quad \text { and } \quad A_{n} \rightarrow \infty .
\end{aligned}
$$

Then (1.1) holds on any $\Omega \subset[0,1]$ with positive measure.

Actually, we will prove that the assumption of Theorem 1 is equivalent to that of the Corollary. Thus, our result extends and unifies previous results. 
Since $a_{n}=o\left(A_{n}\right)$ is necessary for (1.1) (Salem-Zygmund [4]), if we assume (1.8), the condition (1.5) is indispensable for Theorem 1.

The next theorem asserts that (1.8) cannot be weakened.

Theorem 2. Suppose that $\{\lambda(i)\}$ satisfies $\lambda(i)>0$, (1.6) and (1.9). Then there exist sequences $\left\{n_{i}\right\}$ and $\left\{a_{i}\right\}$ satisfying (1.7),

$$
a_{n}=O\left(A_{n} / \lambda(n)\right) \quad \text { and } \quad A_{n} \rightarrow \infty,
$$

such that the central limit theorem (1.1) does not hold on $\Omega=[0,1]$.

Finally, we state a result on the Fourier character of the series. Since (1.11) is always true under Hadamard's gap condition, it includes that of Zygmund [10].

Theorem 3. Let us assume (1.5), (1.6), (1.7), (1.9),

$$
a_{n}=O\left(A_{n} \ell\left(A_{n}\right) / \lambda(n)\right) \text { and } A_{n} \rightarrow \infty,
$$

where $\ell(x)=\sqrt{\log x \log \log x \ldots \log ^{(\gamma)} x}$ and $\gamma \in \mathbf{N}$. Then the series diverges almost surely and is neither a Fourier series nor a Fourier-Stieltjes series.

Before closing the introduction, we note that the same results for lacunary Walsh series can be proved in the same way.

\section{The CEntral Limit theorem}

Let us put $n_{0}=1$ and $\lambda(0)=2 \lambda$, and introduce the following notation:

$$
\begin{aligned}
& p(0)=0, \quad p(k)=\max \left\{i \mid n_{i} \leq 2^{k}\right\}(k \geq 1), \quad l(k)=p(k+1)-p(k), \\
& P(k)=\mathbf{N} \cap(p(k), p(k+1)], \quad \mu(k)=\max _{i \in P(k)} \lambda(i), \quad \nu(k)=\min _{i \in P(k)} \lambda(i) .
\end{aligned}
$$

Since $\left\{n_{i}\right\}$ diverges to infinity, $\{p(k)\}$ does also. If $p(k)+1<p(k+1)$, we have

$$
2>\frac{n_{p(k+1)}}{n_{p(k)+1}}>\prod_{i=p(k)+1}^{p(k+1)-1}\left(1+\frac{c}{\lambda(i)}\right)>1+\sum_{i=p(k)+1}^{p(k+1)-1} \frac{c}{\lambda(i)}>1+c \frac{l(k)-1}{\mu(k)} .
$$

From this and $\mu(k)>\lambda$, it follows that

$$
l(k)=O(\mu(k)) .
$$

By (1.6), we have $\lambda(i)=o(i)$ and $\mu(k)=o(p(k+1))$. Applying this to (2.1) we get

$$
p(k+1) \sim p(k) .
$$

Applying (1.6) and (2.1), we have

$$
0 \leq \frac{\mu(k)-\nu(k)}{\mu(k)} \leq \sum_{i=p(k)}^{p(k+1)-1} \frac{|\lambda(i+1)-\lambda(i)|}{\mu(k)}=o\left(\frac{l(k)}{\mu(k)}\right)=o(1) .
$$

This implies $\mu(k) \sim \nu(k)$, and hence $\mu(k) \sim \lambda(p(k+1))$ and $\mu(k+1) \sim \lambda(p(k+1)+1)$ follow. Since $\lambda(i+1) \sim \lambda(i)$ is clear from (1.5) and (1.6), we have

$$
\mu(k+1) \sim \mu(k) .
$$

The next lemma plays an important role in the proof. 
Lemma 1. For any given integers $j, k, h$, and $q$ satisfying

$$
j<k \text { and } p(j)+1<h \leq p(j+1)<p(k)+1<q \leq p(k+1),
$$

the number of solutions $\left(n_{r}, n_{i}\right)$ of the equation

$$
n_{q}-n_{r}=n_{h}-n_{i} \quad \text { where } p(j)<i<h \quad \text { and } \quad p(k)<r<q,
$$

is at most $2^{j-k+1} \mu(k) / c$.

Proof. If $\left(n_{r}, n_{i}\right)$ is a solution, we have

$$
n_{r}=n_{q}-\left(n_{h}-n_{i}\right)>n_{q}-2^{j}>n_{q}\left(1-2^{j-k}\right) \geq n_{q}\left(1+2^{j-k+1}\right)^{-1} .
$$

Let us denote the least (or greatest) index of $n_{r}$ 's by $m_{1}$ (or $m_{2}$ ). Dividing $n_{q} \geq$ $n_{m_{2}+1}$ by $n_{q}\left(1+2^{j-k+1}\right)^{-1} \leq n_{m_{1}}$, we have

$$
1+2^{j-k+1} \geq \frac{n_{m_{2}+1}}{n_{m_{1}}} \geq \prod_{m=m_{1}}^{m_{2}}\left(1+\frac{c}{\lambda(m)}\right) \geq 1+\frac{c\left(m_{2}-m_{1}+1\right)}{\mu(k)} .
$$

The next lemma can be proved in the same way.

Lemma 2. For any given integers $j, k, h$, and $q$ satisfying

$$
j+1<k \text { and } p(j+1)<h \leq p(j+2)<p(k+1)<q \leq p(k+2),
$$

the number of solutions $\left(n_{r}, n_{i}\right)$ of the equation

$$
n_{q}-n_{r}=n_{h}-n_{i} \quad \text { where } \quad p(j)<i \leq p(j+1) \quad \text { and } \quad p(k)<r \leq p(k+1),
$$

is at most $2^{j-k+2} \mu(k) / c$.

In the proof of Theorem 1 , we assume $\phi_{i}=0$ to simplify notation. The general case can be proved in the same way. We apply the following result ([5]).

Theorem A. Let $\left\{d_{i}\right\}$ be a sequence of real numbers and put

$$
f_{n}(\omega)=\sum_{i=1}^{n} d_{i} \cos 2 \pi i \omega, \quad \Delta_{k}=f_{2^{k+1}}-f_{2^{k}}, \quad D_{n}=\left(\frac{1}{2} \sum_{i=1}^{n} d_{i}^{2}\right)^{1 / 2},
$$

and $B_{k}=D_{2^{k+1}}$. Suppose that the following conditions are satisfied:

$$
\begin{aligned}
& \int_{0}^{1}\left|\frac{1}{B_{n}^{2}} \sum_{k=1}^{n}\left(\Delta_{k}^{2}(\omega)+2 \Delta_{k}(\omega) \Delta_{k+1}(\omega)\right)-1\right| d \omega \rightarrow 0, \\
& B_{n} \rightarrow \infty \text { and } \sup _{\omega \in[0,1]}\left|\Delta_{n}(\omega)\right|=o\left(B_{n}\right) .
\end{aligned}
$$

Then for any $\Omega \subset[0,1]$ with positive measure, the law of $f_{n} / D_{n}$ on $(\Omega, d \omega /|\Omega|)$ converges weakly to the standard normal distribution.

We apply this by putting $\Delta_{k}(\omega)=\sum_{i \in P(k)} a_{i} \cos 2 \pi n_{i} \omega$ and $B_{k}=A_{p(k+1)}$. Let us put $C_{k}=\left\|\Delta_{k}\right\|$ where $\|\cdot\|$ denotes $L^{2}[0,1]$-norm. Obviously we have $C_{k}^{2}=B_{k}^{2}-B_{k-1}^{2}$. By (1.8) and (2.1), we have

$$
\sup _{\omega \in[0,1]}\left|\Delta_{k}(\omega)\right| \leq \sum_{i \in P(k)}\left|a_{i}\right| \leq l(k) \max _{i \in P(k)}\left|a_{i}\right|=o\left(\mu(k) \frac{B_{k}}{\nu(k)}\right)=o\left(B_{k}\right) .
$$


This implies $C_{k}=o\left(B_{k}\right)$ and $B_{k+1}^{2} / B_{k}^{2}=\left(1-C_{k+1}^{2} / B_{k+1}^{2}\right)^{-1}=1+o(1)$. The next estimate follows from the Schwarz inequality:

$$
\sum_{q \in P(k)}\left|a_{q}\right| \leq l^{1 / 2}(k) C_{k}=O\left(\mu^{1 / 2}(k) C_{k}\right) .
$$

To prove (2.4), we divide $\Delta_{k}^{2}$ into three parts; putting

$$
\begin{aligned}
U_{k} & =\frac{1}{2} \sum_{q \in P(k)} a_{q} \sum_{r \in P(k)} a_{r} \cos 2 \pi\left(n_{q}+n_{r}\right) \omega \quad \text { and } \\
V_{k} & =\sum_{p(k)<r<q \leq p(k+1)} a_{q} a_{r} \cos 2 \pi\left(n_{q}-n_{r}\right) \omega,
\end{aligned}
$$

we have $\Delta_{k}^{2}-C_{k}^{2}=U_{k}+V_{k}$. From (2.6), it follows that

$$
\left\|U_{k}\right\| \leq \frac{1}{2} \sum_{q \in P(k)}\left|a_{q}\right| C_{k}=o\left(B_{k} C_{k}\right) \quad \text { and } \quad\left\|V_{k}\right\|=o\left(B_{k} C_{k}\right)
$$

Since $\left\{U_{k}\right\}$ is an orthogonal sequence, we have

$$
\left\|\sum_{k=1}^{n} U_{k}\right\|^{2}=\sum_{k=1}^{n}\left\|U_{k}\right\|^{2}=o\left(B_{k}^{2} \sum_{k=1}^{n} C_{k}^{2}\right)=o\left(B_{k}^{4}\right) .
$$

Noting this and $\left\|\sum\left(U_{k}+V_{k}\right)\right\|^{2} \leq 2\left\|\sum U_{k}\right\|^{2}+2\left\|\sum V_{k}\right\|^{2}$, we have

$$
\left\|\sum_{k=1}^{n}\left(\Delta_{k}^{2}-C_{k}^{2}\right)\right\|^{2}=o\left(B_{k}^{4}\right)+4 \sum_{1 \leq j<k \leq n} \int_{0}^{1} V_{k}(\omega) V_{j}(\omega) d \omega .
$$

In a similar way, we can prove

$$
\left\|\sum_{k=1}^{n} \Delta_{k} \Delta_{k+1}\right\|^{2}=o\left(B_{k}^{4}\right)+4 \sum_{1 \leq j<k \leq n} \int_{0}^{1} W_{k}(\omega) W_{j}(\omega) d \omega
$$

where $W_{k}=\sum_{q \in P(k+1)} a_{q} \sum_{r \in P(k)} a_{r} \cos 2 \pi\left(n_{q}-n_{r}\right) \omega$. By Lemma 1, (1.8) and (2.7), we have

$$
\begin{aligned}
\left|\int_{0}^{1} V_{k}(\omega) V_{j}(\omega) d \omega\right| & \leq \sum_{q \in P(k)}\left|a_{q}\right| \sum_{h \in P(j)}\left|a_{h}\right| \max _{r \in P(k)}\left|a_{r}\right| \max _{i \in P(j)}\left|a_{i}\right| \frac{2^{j-k+1} \mu(k)}{c} \\
& =o\left(B_{k} B_{j} \mu^{1 / 2}(k) \mu^{-1 / 2}(j) 2^{j-k} C_{k} C_{j}\right) .
\end{aligned}
$$


Because of (2.3), for large enough $M$, we have $\mu(k) / \mu(j) \leq M 2^{k-j}$. Hence we have

$$
\begin{aligned}
\sum_{1 \leq j<k \leq n} \int_{0}^{1} V_{k}(\omega) V_{j}(\omega) d \omega & =o\left(B_{n}^{2}\right) \sum_{k=2}^{n} C_{k} \sum_{j=1}^{k-1} \sqrt{2}^{j-k} C_{j} \\
& =o\left(B_{n}^{2}\right) \sum_{k=2}^{n} C_{k}\left(\sum_{j=1}^{k-1} \sqrt{2}^{j-k} C_{j}^{2}\right)^{1 / 2}\left(\sum_{j=1}^{k-1} \sqrt{2}^{j-k}\right)^{1 / 2} \\
& =o\left(B_{n}^{2}\right)\left(\sum_{k=2}^{n} C_{k}^{2}\right)^{1 / 2}\left(\sum_{k=2}^{n} \sum_{j=1}^{k-1} \sqrt{2}^{j-k} C_{j}^{2}\right)^{1 / 2} \\
& =o\left(B_{n}^{3}\right)\left(\sum_{j=1}^{n-1} C_{j}^{2} \sum_{k=j+1}^{n} \sqrt{2}^{j-k}\right)^{1 / 2} \\
& =o\left(B_{n}^{4}\right) .
\end{aligned}
$$

Similarly we have $\sum_{1 \leq j<k \leq n} \int_{0}^{1} W_{k}(\omega) W_{j}(\omega) d \omega=o\left(B_{n}^{4}\right)$. These estimates yield

$$
\left\|\sum_{k=1}^{n}\left(\Delta_{k}^{2}-C_{k}^{2}\right)\right\|=o\left(B_{n}^{2}\right) \quad \text { and }\left\|\sum_{k=1}^{n} \Delta_{k} \Delta_{k+1}\right\|=o\left(B_{n}^{2}\right),
$$

which imply (2.4).

Next we prove the Corollary. Let us put $\rho(i)=1 /\left(n_{i+1} / n_{i}-1\right)$. Let $\Delta x(i)$ denote $x(i+1)-x(i)$.

We now assume $0<\Lambda(i) \rightarrow \infty, \Delta \Lambda(i)=O(1), \rho(i)=o(\Lambda(i))$ and $\rho(i) \leq \Lambda(i)$, and hereafter construct $\lambda(i)$ which satisfies $2 \lambda \vee \rho(i) \leq \lambda(i), \lambda(i)=o(\Lambda(i))$ and $\Delta \lambda(i)=o(1)$. The conditions of Theorem 1 are clearly derived from these. Put $\lambda=\frac{1}{2} \inf _{i} \Lambda(i)$. Let us first construct sequences $1=i_{0}<i_{1}<i_{2}<\cdots$ and $\left\{\Lambda_{0}(i)\right\}$, $\left\{\Lambda_{1}(i)\right\},\left\{\Lambda_{2}(i)\right\}, \ldots$ such that

$$
\begin{gathered}
\rho(i)=o\left(\Lambda_{n}(i)\right) \quad \text { and } \quad \Lambda_{n}(i) \rightarrow \infty \quad(i \rightarrow \infty, n \geq 0), \\
2 \lambda \vee \rho(i) \leq \Lambda_{n}(i) \leq \Lambda_{n-1}(i) \text { and } \Delta \Lambda_{n}(i)=\frac{1}{2} \Delta \Lambda_{n-1}(i) \quad\left(n \geq 1, i \geq i_{n}\right), \\
\Lambda_{n-1}(i) \leq \frac{2}{3} \Lambda_{n-2}(i) \quad\left(n \geq 2, i \geq i_{n}\right) .
\end{gathered}
$$

These sequences are constructed inductively in $n$. First we put $i_{0}=1$ and $\Lambda_{0}(i)=$ $\Lambda(i)$. It is clear that (2.8) is satisfied for $n=0$. After $i_{n-1}$ and $\left\{\Lambda_{n-1}(i)\right\}$ have been constructed, we define $i_{n}$ and $\left\{\Lambda_{n}(i)\right\}$ as follows: We can take $j>i_{n-1}$ such that

$$
\rho(i) \leq \Lambda_{n-1}(i) / 2 \quad \text { and } \quad \Lambda_{n-2}\left(i_{n-1}\right) \leq \Lambda_{n-2}(i) / 3 \quad(i \geq j) .
$$

(The second condition must be omitted in case $n=1$.) Let us take $i_{n} \geq j$ such that $\Lambda_{n-1}\left(i_{n}\right)=\min _{i \geq j} \Lambda_{n-1}(i)$ holds, and define $\Lambda_{n}(i)$ by

$$
\Lambda_{n}(i)= \begin{cases}\Lambda_{n-1}(i), & i<i_{n}, \\ \left(\Lambda_{n-1}(i)+\Lambda_{n-1}\left(i_{n}\right)\right) / 2, & i \geq i_{n} .\end{cases}
$$

By definition, (2.11) holds if we put $j=i_{n}$, and $\Lambda_{n-1}(i) \geq \Lambda_{n}\left(i_{n}\right)$ holds for all $i \geq i_{n}$. Therefore $\Lambda_{n}(i) \leq \Lambda_{n-1}(i)$ holds for $i \geq i_{n}$, and the rest of (2.9) is clear from definition and the first inequality of (2.11). (2.10) follows from the last inequality of (2.11). By definition, (2.8) is clear, and the sequences are well constructed. 
If we put $\lambda(i)=\Lambda_{n}(i)$ for $i_{n} \leq i<i_{n+1}$, it satisfies $2 \lambda \vee \rho(i) \leq \lambda(i), \Delta \lambda(i)=$ $\Delta \Lambda_{n}(i)=\left(\frac{1}{2}\right)^{n} \Delta \Lambda(i)=o(1)$ and $\lambda(i)=\Lambda_{n}(i) \leq \Lambda_{n-1}(i) \leq\left(\frac{2}{3}\right)^{n-1} \Lambda(i)=o(\Lambda(i))$.

Finally, we derive Theorem 1 from the Corollary. By this we see that Theorem 1 and the Corollary are equivalent. We now assume (1.5), (1.6), (1.7) or $\rho(i)=$ $O(\lambda(i))$, and (1.8), and derive the conditions of the Corollary. Conditions (1.5) and (1.8) imply $a_{i} / A_{i} \rightarrow 0$. We can therefore take an increasing sequence $\widetilde{\lambda}(i)$ of positive numbers such that $\tilde{\lambda}(i) a_{i} / A_{i} \rightarrow 0$ and $\Delta \widetilde{\lambda}(i)=o(1)$. If we put $\lambda_{0}(i)=\lambda(i)+\widetilde{\lambda}(i)$, we have $0<\lambda_{0}(i) \rightarrow \infty, \Delta \lambda_{0}(i)=o(1), \rho(i)=O\left(\lambda_{0}(i)\right)$ and $\lambda_{0}(i) a_{i} / A_{i} \rightarrow 0$. Next we construct $1=i_{0}<i_{1}<i_{2}<\cdots$ and $\left\{\lambda_{1}(i)\right\},\left\{\lambda_{2}(i)\right\}, \ldots$ such that $\lambda_{n}(i) \geq \lambda_{n-1}(i)$

$$
\begin{gathered}
0<\lambda_{n}(i) \rightarrow \infty, \quad \Delta \lambda_{n}(i)=o(1), \quad \text { and } \quad \lambda_{n}(i) a_{i} / A_{i} \rightarrow 0 \quad(i \rightarrow \infty, n \geq 0), \\
\left|\Delta \lambda_{n}(i)\right| \leq 1, \Delta \lambda_{n}(i)=2 \Delta \lambda_{n-1}(i), \quad\left|\frac{\lambda_{n}(i) a_{i}}{A_{i}}\right| \leq 1\left(i \geq i_{n}, n \geq 1\right), \\
\lambda_{n-1}(i) \geq \frac{3}{2} \lambda_{n-2}(i) \quad\left(i \geq i_{n}, n \geq 2\right) .
\end{gathered}
$$

It can be achieved first by taking $i_{n+1}$ to satisfy $\lambda_{n}\left(i_{n+1}\right)=\min _{i \geq i_{n+1}} \lambda_{n}(i)$,

$$
\left|\frac{\lambda_{n}(i) a_{i}}{A_{i}}\right| \leq \frac{1}{2}, \quad\left|\Delta \lambda_{n}(i)\right| \leq \frac{1}{2} \quad \text { and } \quad \lambda_{n-1}(i) \geq 2 \lambda_{n-1}\left(i_{n}\right) \quad\left(i \geq i_{n+1}\right),
$$

and then putting $\lambda_{n+1}(i)=2 \lambda_{n}(i)-\lambda_{n}\left(i_{n+1}\right)$ if $i \geq i_{n+1}$ and $\lambda_{n+1}(i)=\lambda_{n}(i)$ otherwise. If we put $\Lambda(i)=\lambda_{n}(i)$ for $n_{i} \leq i<n_{i+1}$, we can verify the conditions on $\Lambda(i)$ in a similar way as before.

\section{Construction of counterexamples}

We may assume $\lambda(i) \rightarrow \infty$, since the condition $a_{n}=o\left(A_{n}\right)$ is necessary for (1.1). There is no loss of generality if we assume $c=1$ and $\lambda(i) \geq 1$. Let us denote by $\|\cdot\|_{\infty}$ the sup-norm on $[0,1]$. Redefine $\{p(k)\}$ by

$$
p(0)=0 \quad \text { and } \quad p(k)=\max \left\{j \mid \sum_{i=1}^{j} \frac{1}{\lambda(i)} \leq k .\right\} \quad(k \geq 1),
$$

and define $l(k), P(k), \mu(k)$ and $\nu(k)$ as before by using new $\{p(k)\}$.

If $p(k)+1<p(k+1)$, we have

$$
\frac{l(k)}{\nu(k)}+\frac{1}{\nu(k+1)}+\frac{1}{\nu(k-1)} \geq \sum_{i=p(k)}^{p(k+1)+1} \frac{1}{\lambda(i)} \geq 1 \geq \sum_{i=p(k)+1}^{p(k+1)} \frac{1}{\lambda(i)} \geq \frac{l(k)}{\mu(k)},
$$

which implies $\liminf l(k) / \nu(k) \geq 1$ and $l(k) \leq \mu(k)$. By using $l(k) \leq \mu(k)$, in the same way as before, we can prove $(2.2), \mu(j) \sim \nu(j)$, and (2.3) in turn. Consequently we have $\mu(j) \sim \nu(j) \sim l(j) \rightarrow \infty$, and therefore we can take $j_{0} \geq 1$ such that $\mu(j) / 2 \leq l(j) \leq 2 \nu(j)$ for $j \geq j_{0}$. We note that

$$
\sum_{j=j_{0}}^{\infty} \frac{1}{l(j)}=\sum_{j=j_{0}}^{\infty} \frac{l(j)}{l^{2}(j)} \geq \sum_{j=j_{0}}^{\infty} \sum_{i \in P(j)} \frac{1}{4 \lambda^{2}(i)}=\infty .
$$

Let us put

$$
a_{i}=\left\{\begin{array}{ll}
1 & \text { if } i \leq p\left(j_{0}\right), \\
A_{p(j)} / l(j) & \text { if } i \in P(j), j \geq j_{0},
\end{array} \quad \text { and } \quad b_{j}=a_{p(j+1)},\right.
$$


and define $\Delta_{k}$ as before. We easily have $A_{p\left(j_{0}\right)}>0, a_{i}=O\left(A_{i} / \lambda(i)\right)$ and

$$
A_{p(k+1)}^{2}=A_{p\left(j_{0}\right)}^{2} \prod_{j=j_{0}}^{k}\left(1+\frac{1}{2 l(j)}\right) \geq \frac{A_{p\left(j_{0}\right)}^{2}}{2} \sum_{j=j_{0}}^{k} \frac{1}{l(j)} \rightarrow \infty .
$$

Let us take an increasing sequence $\{q(j)\}$ of integers such that

$$
q\left(j_{0}\right)=p\left(j_{0}\right)+1 \quad \text { and } \quad 2^{q(j+1)-q(j)} \geq \max \left\{2 l(j+1), \pi A_{p(j)} l^{2}(j) j^{2}\right\},
$$

and introduce $\left\{n_{i}\right\}$ by

$$
n_{p(j)+l}= \begin{cases}2^{p(j)+l} & \text { if } j<j_{0}, 1 \leq l \leq l(j), \\ 2^{q(j)} l & \text { if } j \geq j_{0}, 1 \leq l \leq l(j) .\end{cases}
$$

If $j \geq j_{0}$ and $1 \leq l \leq l(j),(1.7)$ is verified by noting $l(j) \leq 2 \nu(j) \leq 2 \lambda(p(j)+l)$;

$$
\frac{n_{p(j)+l+1}}{n_{p(j)+l}} \geq 1+\frac{1}{l(j)} \geq 1+\frac{1}{\lambda(p(j)+l)} \quad \text { and } \quad \frac{n_{p(j)+1}}{n_{p(j)}}=\frac{2^{q(j)-q(j-1)}}{l(j)} \geq 2 .
$$

By using the Dirichlet kernel, we see that there exists an absolute constant $c_{0}>0$ such that for all integers $m$ and $l, P\left(\left|\sum_{j=1}^{l} \cos 2 \pi m j \omega\right|>l / e\right) \geq c_{0} / l$. Applying this, we have $P\left(\left|\Delta_{j}\right| \geq b_{j} l(j) / e\right) \geq c_{0} / l(j)$. Note that $b_{j} l(j) / e=A_{p(j)} / e$ for $j \geq j_{0}$. If we put $J_{m}=\left\{j=j_{0}, \ldots, m \mid e A_{p(j)} \geq A_{p(m+1)}\right\}$, we have

$$
\sum_{j=1}^{m} P\left(\left|\Delta_{j}\right| \geq \frac{A_{p(m+1)}}{e^{2}}\right) \geq \sum_{j \in J_{m}} P\left(\left|\Delta_{j}\right| \geq \frac{A_{p(j)}}{e}\right) \geq \sum_{j \in J_{m}} \frac{c_{0}}{l(j)} .
$$

Since we have $A_{p(m+1)} / A_{p(j)} \leq \exp \left(\frac{1}{2} \sum_{k=j+1}^{m} 1 / l(k)\right)$, by putting $J_{m}^{\prime}=\{j=$ $\left.j_{0}, \ldots, m \mid \sum_{k=j+1}^{m} 1 / l(k) \leq 2\right\}$, we have $J_{m}^{\prime} \subset J_{m}$ and hence

$$
\sum_{j=1}^{m} P\left(\left|\Delta_{j}\right| \geq \frac{A_{p(m+1)}}{e^{2}}\right) \geq \sum_{j \in J_{m}^{\prime}} \frac{c_{0}}{l(j)} \rightarrow 2 c_{0}
$$

Let $\omega=\sum_{r=1}^{\infty} 2^{-r} d_{r}(\omega)$ be the dyadic expansion of $\omega$, and put

$$
\widetilde{X}_{j}(\omega)=\sum_{i \in P(j)} a_{i} \cos 2 \pi n_{i}\left(\sum_{r=q(j)+1}^{q(j+1)} 2^{-r} d_{r}(\omega)\right) \text { and } X_{j}=\widetilde{X}_{j}-E \widetilde{X}_{j} .
$$

Clearly, $\left\{X_{j}\right\}$ is an independent sequence. Because of $E \Delta_{j}=0$ and the estimate

$$
\left\|\Delta_{j}-\widetilde{X}_{j}\right\|_{\infty} \leq b_{j} \sum_{i \in P(j)} 2 \pi n_{i} 2^{-q(j+1)} \leq \pi b_{j} l^{2}(j) 2^{q(j)-q(j+1)} \leq 1 / j^{2},
$$

we have $\left\|\Delta_{j}-X_{j}\right\|_{\infty} \leq 2 / j^{2}$. If we put $\sigma_{j}^{2}=E X_{j}^{2}$ and $s_{m}^{2}=\sum_{j=1}^{m} \sigma_{j}^{2}$, we get

$$
\left|s_{m}-A_{p(m+1)}\right| \leq \sum_{j=1}^{m}\left\|\Delta_{j}-X_{j}\right\|_{\infty} \leq 4 \quad \text { and } \quad\left|\sigma_{j}-E^{1 / 2} \Delta_{j}^{2}\right| \leq \frac{2}{j^{2}} .
$$

Combining these with (3.1) and $E \Delta_{j}^{2}=A_{p(j)}^{2} / 2 l(j)$, we have

$$
s_{m} \rightarrow \infty \quad \text { and } \quad \sigma_{m}=O\left(1 / m^{2}\right)+E^{1 / 2} \Delta_{m}^{2}=o\left(A_{p(m)}\right)=o\left(s_{m}\right) .
$$


If the central limit theorem (1.1) holds, then it holds for $\left\{X_{i}\right\}$. The Lindeberg theorem claims that (cf. Chapter XV of Feller [2]) under (3.3), the central limit theorem $s_{m}^{-1} \sum_{i=1}^{m} X_{k} \stackrel{\mathcal{D}}{\longrightarrow} N_{0,1}$ implies the Lindeberg condition

$$
s_{m}^{-2} \sum_{i=1}^{m} E\left(X_{i}^{2}:\left|X_{i}\right|>\varepsilon s_{m}\right) \rightarrow 0 \text { for all } \varepsilon>0 .
$$

From this we have

$$
\lim _{m \rightarrow \infty} \sum_{i=1}^{m} P\left(\left|X_{i}\right|>\varepsilon s_{m}\right)=0 \text { for all } \varepsilon>0 .
$$

On the other hand, by (3.2) we have

$$
0<c_{0} \leq \sum_{i=1}^{m} P\left(\left|\Delta_{i}\right|>A_{p(m+1)} / e^{2}\right) \leq \sum_{i=1}^{m} P\left(\left|X_{i}\right|>s_{m} / e^{3}\right)
$$

for large $m$. These contradict each other.

\section{Fourier CHARACTER OF LACUNARY SERIES}

We prove Theorem 3 for $\gamma=2$. The general case can be proved by iterating the following argument $\gamma$ times. Let us put $S_{n}(\omega)=\sum_{i=1}^{n} a_{i} \cos \left(2 \pi n_{i} \omega+\phi_{i}\right)$. We may assume $\delta=\sup _{i}\left|a_{i}\right|<\infty$, otherwise the conclusion is clear. Let us put

$$
r(0)=0 \quad \text { and } \quad r(m)=\max \left\{i \mid A_{i} \leq \delta m\right\} \quad(m \geq 1) .
$$

By $\delta^{2} m^{2} \geq A_{r(m)}^{2}=A_{r(m)+1}^{2}-a_{r(m)}^{2} / 2>\delta^{2}\left(m^{2}-1 / 2\right)$, we have $A_{r(m)} \sim \delta m$, $A_{r(m)}^{2}-A_{r(m-1)}^{2} \sim 2 \delta^{2} m$ and $A_{r(m)}^{-1}-A_{r(m-1)}^{-1}=O\left(m^{-2}\right)$.

First we assume that $\left\{S_{n}\right\}$ converges on some set $E$ with $|E|>0$ and derive a contradiction. Let us put $b_{i}=a_{i} / A_{i}, B_{m}=\frac{1}{2} \sum_{i=1}^{m} b_{i}^{2}$ and $T_{m}(\omega)=\sum_{i=1}^{m} b_{i} \cos 2 \pi n_{i} \omega$. Since each term of $T_{m}$ is a product of the term of $S_{m}$ and non-negative decreasing sequence $1 / A_{i}$, by using the Abel's Theorem (cf. (2.4) of Chapter I in Zygmund [11]), $\left\{T_{n}\right\}$ converges on $E$. Since we have

$$
B_{r(m)}^{2}=\sum_{k=1}^{m} \sum_{i=r(k-1)+1}^{r(k)} \frac{a_{i}^{2}}{2 A_{i}^{2}} \sim \sum_{k=1}^{m} \frac{A_{r(k)}^{2}-A_{r(k-1)}^{2}}{A_{r(k)}^{2}} \sim \sum_{k=1}^{m} \frac{2}{k} \sim \log m^{2} \sim \log A_{r(m)}^{2},
$$

we get $B_{k}^{2} \sim \log A_{k}^{2}$, and hence we can prove

$$
b_{i}=o\left(B_{i} \sqrt{\log B_{i}} / \lambda(i)\right) \text { and } \quad B_{i} \rightarrow \infty .
$$

Let us repeat the above argument, i.e., take a sequence $\left\{r^{\prime}(m)\right\}$ as $B_{r^{\prime}(m)} \sim m$ and define $c_{i}=b_{i} / B_{i}, C_{k}=\frac{1}{2} \sum_{i=1}^{k} c_{i}^{2}$ and $Z_{m}(\omega)=\sum_{i=1}^{m} c_{i} \cos 2 \pi n_{i} \omega$. In the same way we can prove that $C_{k}^{2} \sim \log B_{k}^{2}$ and hence

$$
c_{i}=o\left(C_{i} / \lambda(i)\right) \text { and } C_{i} \rightarrow \infty,
$$

and that $Z_{\infty}$ converges on $E$ and thereby $Z_{m} / C_{m} \rightarrow 0$ on $E$. By (4.2) we can apply Theorem 1 and conclude that $Z_{m} / C_{m}$ converges to $N_{0,1}$ on $E$, which is a contradiction.

Next we assume that the series is a Fourier series or a Fourier-Stieltjes series. Let us take $\rho \in(1 / 2,1)$. By the Riesz-Kolmogorov inequality (cf. (6.8) or (6.27) of Chapter VII in Zygmund [11]), we have $E\left|S_{m}\right|^{\rho}=O(1)$. 
Let us redefine $b_{i}$ as $b_{i}=a_{i} / A_{r(m+1)}$ if $r(m)<i \leq r(m+1)$, and define $B_{k}$ and $T_{m}$ as before by using these $b_{i}$. In a similar way, we can prove $B_{k}^{2} \sim \log A_{k}^{2}$ and (4.1). For any $m$, let us take $n$ such that $r(n)<m \leq r(n+1)$. By applying the Abel's partial summation (the Abel transformation) to $T_{m}=\left(S_{m}-S_{r(n)}\right) / A_{r(n+1)}+$ $\sum_{k=1}^{n}\left(S_{r(k)}-S_{r(k-1)}\right) / A_{r(k)}$, we have

$$
\begin{aligned}
E\left|T_{m}\right|^{\rho} & =E\left|\frac{1}{A_{r(n+1)}} S_{m}+\sum_{k=1}^{n}\left(\frac{1}{A_{r(k)}}-\frac{1}{A_{r(k+1)}}\right) S_{r(k)}\right|^{\rho} \\
& \leq \frac{1}{A_{r(n+1)}^{\rho}} E\left|S_{m}\right|^{\rho}+\sum_{k=1}^{n}\left(\frac{1}{A_{r(k)}}-\frac{1}{A_{r(k+1)}}\right)^{\rho} E\left|S_{r(k)}\right|^{\rho} .
\end{aligned}
$$

Since $\left(\frac{1}{A_{r(k)}}-\frac{1}{A_{r(k+1)}}\right)^{\rho}=O\left(k^{-2 \rho}\right)$ is summable in $k$, we have $E\left|T_{m}\right|^{\rho}=O(1)$.

Let us redefine $c_{i}$ as $c_{i}=b_{i} / B_{r^{\prime}(m+1)}$ if $r^{\prime}(m)<i \leq r^{\prime}(m+1)$, and define $C_{k}$ and $Z_{m}$ as before. Then we have (4.2) and $E\left|Z_{m}\right|^{\rho}=O(1)$. Thus $Z_{m} / C_{m}$ converges to $N_{0,1}$ in law, and to 0 in $L^{\rho}$-sense and hence in probability. This is a contradiction.

\section{REFERENCES}

1. P. Erdős, On trigonometric series with gaps, Magyar Tud. Akad. Mat. Kutato Int. Közl. 7 (1962), 37-42. MR 26:2797

2. W. Feller, An introduction to probability theory and its applications vol. II, second ed., Wiley, New York, 1971. MR 42:5292

3. A. N. Kolmogorov, Une contribution à l'étude de la convergence des séries de Fourier, Fund. Math. 5 (1924), 26-27.

4. R. Salem \& A. Zygmund, On lacunary trigonometric series, Proc. Nat. Acad. Sci. 33 (1947), 333-338. MR 9:181d

5. S. Takahashi, A version of the central limit theorem for trigonometric series, Tôhoku Math. J. 16 (1964), 384-398. MR 30:4114

6. _ On trigonometric series with gaps, Tôhoku Math. J. 17 (1965), 227-234. MR 32:4459

7. _ On lacunary trigonometric series, Proc. Japan Acad. 41 (1965), 503-506. MR 33:4564

8. _ On the lacunary Fourier series, Tôhoku Math. J. 19 (1967), 79-85. MR 35:2067

9. _ On lacunary trigonometric series II, Proc. Japan Acad. 44 (1968), 766-770. MR 39: 4583

10. A. Zygmund, On the convergence of lacunary trigonometric series, Fund. Math. 16 (1930), 90-97; Correction, Fund. Math. 18 (1932), 312.

11. MR 21:6498

Department of Mathematics, Kobe University, Rokko, Kobe, 657 Japan

E-mail address: fukuyama@math.s.kobe-u.ac.jp

YAMAMICHI-CHO 21-1-601, HiROSAKI, 036 JAPAN 